Document downloaded from:

http://hdl.handle.net/10251/44015

This paper must be cited as:

Heras Barberá, SM.; Julian Inglada, VJ.; Botti Navarro, VJ. (2010). Applying CBR to manage argumentation in MAS. International Journal of Reasoning-based Intelligent Systems. 2(2):110-117. doi:10.1504/IJRIS.2010.034906.

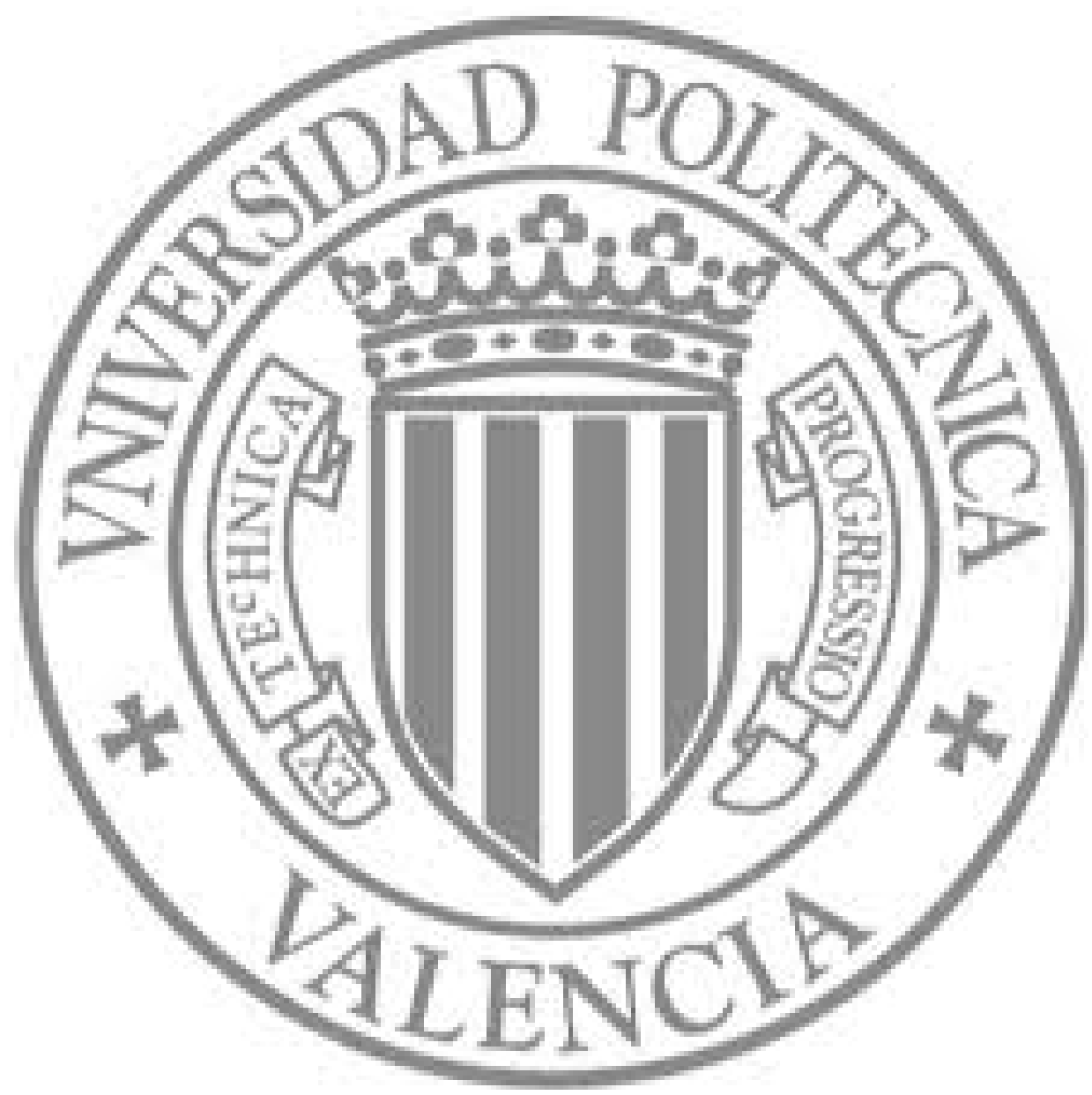

The final publication is available at

http://dx.doi.org/10.1504/IJRIS.2010.034906

Copyright Inderscience 


\title{
Applying CBR to Manage Argumentation in MAS
}

\author{
Stella Heras, Vicente Julián, Vicente Botti \\ Departamento de Sistemas Informáticos y Computación, \\ Universidad Politécnica de Valencia, Camino de Vera s/n 46022, Valencia, (Spain) \\ Tel. (+34) 963877350 - Fax. (+34) 963877359 \\ Email: \{sheras, vinglada, vbotti\}@dsic.upv.es
}

\begin{abstract}
The application of argumentation theories and techniques in multi-agent systems has become a prolific area of research. Argumentation allows agents to harmonize two types of disagreement situations: internal, when the acquisition of new information (e.g. about the environment or about other agents) produces incoherences in the agents' mental state; and external, when agents that have different positions about a topic engage in a discussion. The focus of this paper is on the latter type of disagreement situations. In those settings, agents must be able to generate, select and send arguments to other agents that will evaluate them in their turn. An efficient way for agents to manage these argumentation abilities is by using case-based reasoning, which has been successfully applied to argumentation from its earliest beginnings. This reasoning methodology also allows agents to learn from their experiences and therefore, to improve their argumentation skills. This paper analyses the advantages of applying case-based reasoning to manage arguments in multi-agent systems dialogues, identifies open issues and proposes new ideas to tackle them.
\end{abstract}

Keywords: argumentation; case-based reasoning; multi-agent systems.

\section{Introduction}

Argumentation Theory (AT) and Artificial Intelligence (AI) have a long history of mutual contributions. However, the application of argumentation to the Multi-Agent Systems (MAS) paradigm is a recent trend that has gained an increasing interest during the last 10 years (Bench-Capon et al., 2007). In settings where autonomous agents dynamically perceive changes in their environment (e.g. about the environment itself or about other agents), there is a need for a way for resolving the potential incoherences that these changes can cause in the agents' mental state. Furthermore, as agents act as individual entities, conflicts of opinion with other agents are likely to appear. Argumentation has been adopted in the MAS community as a natural way for harmonising these disagreement situations. Its techniques can be used to specify autonomous agent reasoning and to facilitate multi-agent interaction (Rahwan, 2005).

Case-Based Reasoning (CBR) (Aamodt and Plaza, 1994) is other important AI research field where argumentation-based techniques have a broad history of successful applications. CBR systems solve new problems on the basis of the assumption that similar problems have similar solutions. This assumption also applies to Anglo-American law systems, whose judicial standard 'stare decisis' establishes that similar cases must be resolved with similar verdicts. Thus, most seminal applications of CBR worked on those domains and were developed to support attorneys in their arguments over the interpretation of precedent cases. The first case-based system for legal reasoning was HYPO (Ashley, 1991), which cited precedent cases as ' $3-p l y$ ' legal arguments to decide the winner of a trial. Afterwards, the HYPO argumentation model was extended by CATO (Aleven et al., 1997), an intelligent learning environment for teaching law students to build arguments from precedent cases. Although CATO was initially developed to work in the same domain as HYPO (i.e. trade secrets American law), its argumentation model was subsequently adopted by several systems that operated in diverse domains. BankXX (Rissland et al., 1993), a case-based legal system for retrieving useful information in the domain of bankruptcy American law is an example.

Contemporary to HYPO was Sycara's PERSUADER system (Sycara, 1990), which constitutes the fist step towards the application of CBR to manage argumentation issues in multi-party discussions. PERSUADER was the first system that integrated concepts of CBR and AT to create a negotiation model for a mediator agent that controls a dialogue between agents. In this system, the role of the mediator is to negotiate with two agents representing a company and its trade union and persuade them to accept the terms of a specific labour agreement (e.g. a contract over salaries, holidays or pensions). Opposite to most similar systems of its time, PERSUADER does not assume that the company and the trade union are 
cooperative by default, but it deals with argumentation when both parties try to derive the maximum own benefit from the negotiation. Therefore, persuading a party implies to change its individual assessment about the contract under discussion. In PERSUADER, a possible way of bringing about such a change is by using $\mathrm{CBR}$ to retrieve precedents that demonstrate that the terms of the current contract were considered acceptable for this party or for similar parties in the past. Although Sycara's system paved the way for hybrid applications of CBR to multi-agent argumentation, it cannot be viewed as a today's MAS. The agent's model was somehow specified by abstractions and the correspondence between what is considered 'agent' in PERSUADER and in the current agents technology is quite fuzzy. However, the system was a foundational framework that outlined the potential advantages of applying CBR to argumentation in MAS.

The good results obtained by PERSUADER and the CBR systems applied to legal argumentation suggest that this reasoning methodology is particularly suitable for reasoning about arguments in MAS dialogues. In settings where a group of agents try to reach agreements by using argumentation techniques in their dialogue, an agent can be persuaded by other agents to accept their arguments. These arguments can provide an agent with new information that could produce changes in the agent's point of view and hence, in the knowledge that the agent has about himself, the environment or its neighbours. Cases can store incomplete, uncertain and inconsistent information that can be updated afterwards in the revision and retention phases of the CBR cycle. Therefore, reasoning with knowledge that has been stored in the form of cases facilitates later revisions and updates. Moreover, CBR also allows agents to learn from experiences and improve their argumentation skills.

This paper analyses the advantages that have been achieved by applying CBR to manage arguments in dialogues between agents. Specific examples of systems that incorporate CBR facilities for arguing in MAS are also provided. Although the number of systems that follow this hybrid approach is still scarce, the important contributions that they have made to the argumentation in MAS foresee a promising new area of research. Section 2 attempts to clarify the advances achieved in this area and to identify open issues that need from further research. In addition, some preliminary ideas to face these challenges are also proposed. Section 3 briefly introduces how reasoning with previous experiences can influence the interaction between agents. Finally, section 3 summarises the main conclusions drawn from the analysis performed in this paper.

\section{Case-Based Argument Management}

Currently, the type of arguments that agents interchange are very influenced by the type of dialogue that they are engaged in. The argumentation theorists Walton and Krabbe defined a famous typology of dialogues that classifies them depending on their initial situation, their main goal and the aims of the participants (Walton and Krabbe, 1995). This typology includes dialogues for: persuasion, negotiation, information inquiry, deliberation and eristics. The different types of dialogues between agents have been commonly classified in accordance with Walton and Krabbe's typology. Although the authors themselves also considered mixed dialogues and, as it was argued in (Parsons et al., 1998), in a high level view of multi-agent interaction the elements that characterize these dialogues can intermingle, for our purposes this basic classification clarifies enough the final objective of the dialogue.

Following Walton and Krabbe's typology, the main research done to apply CBR to manage arguments in MAS can be classified into systems for collaborative deliberation and for argumentationbased negotiation. This classification determines how arguments are represented and managed in each specific system. However, enhancing MAS with CBR capabilities has provided many advantages for argument management in every case. On one hand, the case-base can store information that can be used afterwards to generate, select and evaluate arguments. On the other hand, reasoning about cases can improve the argumentation skills of agents. Next sections explain these advantages, showing examples of systems that address different types of dialogues, and propose open issues for future research.

\subsection{Argument Storage}

Since the knowledge about past experiences is the reasoning basis of CBR systems, the specific contents and structure of the case-base is an important design decision that differentiates and defines their operation. Therefore, the contents of the cases depend on the objective that is pursued with the system. In MAS the case-base is often used as knowledge resource and represents the 'memory' of the agents or of the system itself, like a track record storing the experience gained along their life. This knowledge 
increases agents autonomy and may lead to a more successful interaction between them. This section shows some examples of how relevant information for arguing can be stored in the form of cases and introduces how enhancing the contents and the structure of the case-base can result in a more efficient use of that information.

In argumentation-based collaborative deliberation frameworks, the role of CBR depends on the agent capabilities, which also determine the objective of the collaboration. On one hand, there are frameworks where the objective of the system is to moderate the dialogue of a group of agents, which have not enough autonomy to independently solve a problem. The agents must collaborate to be able to achieve a coherent joint decision via argumentation. Here, CBR can be used to generate, select or evaluate the arguments submitted by the agents and to help the system in directing the interaction between them. In this kind of frameworks, the agents can interact either directly or centralise the interaction into a mediator agent (MA). A preliminary example of the former type was the collaborative decision maker HERMES, which integrated CBR and argumentative based reasoning into its Argument Builder Tool (Karacapilidis et al., 1997, 2001). The system helps a group of human agents to argue in supporting or attacking alternative positions in a decision process. HERMES is an Argumentation-based Decision Support System (ADSS) that maps the argumentation process to a discussion graph with a tree structure. In this framework, the system controls the interaction between agents and uses CBR as a reasoning component that provides warrants to the agents' arguments by referring to previous disputes. There is a common case-base where the cases are flexible entities with store a set of argumentation elements that can be interpreted depending on the state of the discourse (by using the previous instances of the discussion graph) and the agent viewpoints. A viewpoint can be shared by many agents and represents the interests of the agent by means of a list of predicates and the inference schema that the agent has adopted in the discussion.

A current example of collaborative deliberation frameworks is the multi-agent decision support system CARREL+ (Vázquez-Salceda et al., 2003), whose architecture has been extended with the new argument-based selection model ProClaim (Tolchinsky et al., 2006). In CARREL+ an agent that represents an organ donor (DA) and a set of agents that represent potential recipients (RA's) argue about the viability of the organ transplantation to any recipient. Otherwise the organ is discarded. Opposite to HERMES, the interaction in ProClaim is centralised in a MA that controls the deliberation. CBR is used to evaluate the arguments about organ viability that the agents generate in basis of the knowledge acquired from past transplants. To this end, the MA uses a case-base where each case has associated a case description, which represents the medical information describing the transplant and an argument graph, which shows the arguments that were submitted by the agents engaged in that past transplant process. Moreover, an argument graph has an evidential support, which is a tuple $<\mathrm{F}, \mathrm{K}>$ where $\mathrm{F}$ represents the certainty in the decision correctness and $\mathrm{K}$ the number of cases that share the argument graph. Recently, ProClaim model has also been successfully applied to other systems for agent deliberation: a system for wastewater management in river basins (Aulines et al., 2007) and a system for deliberation over action proposals (Tolchinsky et al., 2007).

On the other hand, if the agents are completely autonomous and are able to obtain a solution for the issue under discussion without any help, CBR may be used to solve the problem independently. This is the approach adopted in the Argumentation-based Multi-Agent Learning framework (AMAL), where the agents can act as independent classifiers (Ontañón and Plaza, 2007) that have private case-bases and learn from their experiences. The argumentation-based interaction in this case is intended to improve the solution quality by adding the knowledge of a set of expert agents. If the knowledge of two agents gives rise to contradictory solutions, $\mathrm{CBR}$ is used to choose the preferred one. In this framework there is a predefined set of solution classes. Therefore, each case $\langle P, S\rangle$ is a tuple that contains a case description (i.e. the set of attributes that describe the problem $P$ ) and the solution class $S$ where that case was classified.

In the case of argumentation-based negotiation dialogues, CBR has been applied to negotiate more efficiently by using the experience obtained from past negotiations. This is the approach of the Casebased Reflective Negotiation model (Soh and Tsatsoulis, 2001, 2005). In their framework a group of situated agents that control certain sensors try to track mobile targets. The sensors have limited coverage and power capabilities and hence, the agents must negotiate to track their objectives. This is a resource allocation problem where the agents are cooperative in principle, but they also try to meet their own objectives. When a new negotiation occurs, the agents determine the best negotiation strategy to argue with its partners. To this end, they use the knowledge about past negotiation encounters that is stored in their case-bases. Since agents can play two different roles, as initiators or responders of the negotiation, in this framework the case-base is divided into two independent case-bases. Therefore, each case-base contains specialised information either about negotiations that the agent initiated in the past or about 
negotiations that other agents held with it. In both case-bases the cases include descriptors, which are parameters that determine if a current negotiation is similar to that represented in the case and a set of negotiation parameters, which define the main characteristics of that past negotiation. However, the specific case descriptors and negotiation parameters are different in each case-base.

\section{Open Issues}

In any CBR system, the way in which the information is represented in the case-base determines the reasoning techniques that will be able to process that information. In argumentation domains, how to reason about arguments, their interpretation and the relations between them are key issues that must be dealt with. Therefore, in order to ease the reasoning over the contents of the case-base such issues must be considered in its design process. To date, most hybrid systems that incorporate CBR capabilities to argue in MAS store the information of the cases in simple structures of the form 'attribute-value'. Commonly, this information is used to compute the similarity between the current situation in the dialogue and a potential precedent. Although some of them also enrich this information by relating the case with the specific stage of the argumentation dialogue that it represents, the implementations are very dependent of the particular architecture and system domain and the semantic relations between arguments are not considered. If the arguments are simply stored as data in the cases, the semantic knowledge that has been acquired during the dialogue is wasted. In addition, to provide the cases with general domain knowledge would also help to their interpretation. For instance, objective conventions about the domain could be incorporated in the cases and used afterwards to interpret arguments. Furthermore, storing the cases with knowledge intensive structure (i.e. using Knowledge Intensive CBR (KI-CBR) (Aamodt, 2004) instead of classical plain representations) would allow agents to compute similarities by reasoning about semantic criteria instead of reasoning only about their syntactic properties. How argumentation-based interactions can profit from the properties of KI-CBR is still an open issue that researchers must investigate.

The representation and manipulation of commitments in different types of dialogues is also an open issue that needs from more research. During the interaction, agents may need to contract commitments to reach mutually beneficial agreements. $\mathrm{CBR}$ can be useful to store and manage such commitments. For instance, the CBR cycle can be used to decide if an agent should contract a commitment that could come into conflict with previous commitments. Reasoning about past commitments can be also useful to determine, in view of the final result obtained from the fulfilment of past commitments, if contracting a similar commitment is really worth. Other interesting use of CBR can be to provide warrants about the agent capability to make a commitment by showing similar commitments that it kept in the past.

Finally, case-base consistency matters are also important issues that must be coped with in case-based argumentation in MAS. These systems are highly dynamic and a change in the environment or the input/output of agents in the dialogue can make the case-base contents to become obsolete. In settings where the case-base information is used to generate arguments, a deficient maintenance of the case-base could cause the system to infer incoherences

\subsection{Argument Generation, Selection and Evaluation}

An essential element that differentiates argumentation-based multi-agent frameworks from conventional frameworks is their ability to generate arguments, to select the best outgoing argument from the set of candidates and to evaluate incoming arguments. Arguments can be information about the agent's state, about the world or about the target of the discussion that an agent send to another to persuade it to accept its position about a specific issue under discussion.

This information may be generated by reusing the knowledge stored in the agent's case-base. This is the approach used in the Case-based Reflective Negotiation model. The arguments of this framework are pieces of information that the initiator agent sends to the responder agent in order to surpass its resource negotiation threshold and convince the agent to share it. The CBR manager obtains them by comparing the cases descriptors and with the features of the current situation and retrieving the most similar case from the case-base. Then, the negotiation strategy that will follow the agent is deduced from the negotiation parameters of that case. This negotiation strategy specifies which type of information is most effective to persuade a particular responder agent (i.e. information about the world, about the neighbours or about the agent itself).

Following the same approach, the AMAL framework use both specific cases and justified predictions as information pieces to generate three types of arguments: justified predictions, counterarguments and counterexamples. A justified prediction is a tuple $<A, P, S, D>$ which is generated by the agent $A$ to specify 
that it believes that the correct solution for the problem $P$ is $S$ and the evidence that supports this decision is $D$. The meaning of $D$ is that all or most of the cases that are similar to $P$ in the case-base belong to the predicted solution $S$. A justified prediction can be generated using CBR in combination with any learning method, like decision trees or Lazy Induction of Descriptions (LID) (Armengol and Plaza, 2001). Currently, the AMAL framework uses the LID method to generate justified predictions. A counterargument is a justified prediction $\left\langle A, P, S^{\prime}, D^{\prime}\right\rangle$ that generates an agent to rebut an argument generated by other agent. This counterargument shows its evidence $D^{\prime}$ for believing that the correct solution $S$ ' of the problem $P$ is different than the initially proposed solution $S$. Finally, a counterexample is a case that an agent can send to support a counterargument. In other words, a counterexample is a case from its case-base that classifies $P$ as belonging to a different solution $S$ '.

A different way to generate arguments is to explicitly create them by using some logic language and argumentation theory concepts, such as argumentation schemes and critical questions. These schemes represent stereotyped patterns of everyday reasoning that have been adapted in MAS to model argumentation dialogues between agents. In argumentation schemes, the arguments take the form of a set of inference rules by which, given a set of premises, a conclusion can be inferred. The argumentation schemes of Walton (Walton, 1996) and their underlying extensions are the most widely used in MAS. Walton's schemes have a set of critical questions attached, which represents potential attacks that refute the conclusion supported by the scheme. This feature very clarifies the possible movements of the argumentation and constitutes a desirable advantage for structuring the dialogue between agents. An example of a framework that uses this approach is CARREL+, which characterises completely the space of possible arguments in its Argument Scheme Repository (ASR) (Tolchinsky et al. 2006b). Arguments in CARREL + are instantiations of the argument schemes and critical questions of the ASR. The agents build arguments by using the fist order logic programming language proposed in (Modgil et al. 2005). Although CBR is not directly used here to generate arguments, the argumentation schemes determine the structure of the dialogue (and hence the possible moves on the argumentation graphs of the case-base). Therefore, the case-base of the MA is structured by means of these argument schemes and critical questions.

Once the candidate outgoing arguments have been generated, the next step is to select the best argument to achieve the agent's objectives. A typical approach for selecting arguments is to rank them with respect to some preference order. In the Case-Based Reflective Negotiation model, for instance, the CBR manager of each agent uses domain specific rules to order its arguments from more to less usefulness to convince the responder agent in each step of the negotiation. This process can also be done concurrently with the argument generation, implicitly with the reasoning cycle of the CBR system, as it is done in the AMAL framework. A different approach is followed by HERMES. In this system, agents generate arguments by hand and submit them to the system by instantiating some of the discourse acts that HERMES has pre-established. Afterwards, the Argument Builder Tool of the system uses CBR to retrieve the cases whose target coincides with the agent's current argumentation objective. Then, the system performs a case selection based on the agents' point of view and the current state of the argumentation and uses those cases to provide precedent warrants to the agents' arguments.

Finally, the arguments submitted must be evaluated in order to decide whether they are accepted and thus, the receiver has been persuaded. To perform the evaluation, either objective or subjective considerations can be borne in mind (Rahwan et al. 2003). The former represent shared conventions about the world whereas the latter stand for agents' individual intentions and preferences. Combining both considerations, in the Case-based Reflective Negotiation model the responder agent retrieves the best negotiation strategy from its responder case-base and adapts the past negotiation experience using a relaxed constraint satisfaction approach. This process is performed by comparing the cases' descriptors with the features of the current negotiation. Then, the responder agent uses the information gathered from the negotiation parameters of the most similar case to evaluate the evidence support of the arguments (pieces of information) that it has received.

ProClaim is an example of framework that performs an objective evaluation of arguments. The MA of this model uses its objective considerations, which are based on previous similar dialogues, to evaluate the resulting argument graph. If there are arguments that attack each other, it must decide the winning arguments. To perform this task, the MA compares the argument graph with those graphs stored in its case-base and retrieves the similar ones. Then, it rejects the argument graphs that contain non-applicable arguments by using the case descriptions and those whose evidential support falls below some threshold. The resulting set of graphs is merged into a solution graph that constitutes the CBR proposed decision about the viability of an organ transplantation. This solution graph provides evidential support to 
determine the winning arguments and thus, the decision validity of the current argument graph. Furthermore, it can also propose possible new arguments that could apply to the current situation.

Finally, an example of subjective evaluation of arguments is developed in the AMAL framework, where two contradicting arguments are evaluated for the agents who generated them in accordance with a preference relation. This relation is a subjective criteria based on a confidence measure that is calculated for each justified prediction. To compute the case-based confidence, the number of cases in the case-base that supports the justified prediction and the number of them which are counterexamples is determined. The more the supporting cases, the higher the confidence. The winning argument is that with the highest confidence.

\section{Open Issues}

To date, the argument generation, selection and evaluation processes are very dependent on the specific design decisions of each framework. However, CBR can be used to provide more standardised ways to manage arguments in MAS. Over the last years, the power of explanations to make the reasoning process more understandable has gained an increasing interest in the CBR research community (Sørmo et al., 2005). The CBR explanation techniques can be applied to generate and evaluate arguments in MAS. For instance, explanations could be used to generate arguments that support a specific solution derived from the agent case-base. Some preliminary work was done in the AMAL framework, but it only applies to classification domains. If explanations are provided, argument evaluation can be performed by checking if the reasoning process that generated an argument was correct. Other interesting improvement that can be further investigate if arguments are stored in cases by following a knowledge intensive approach is to explore the semantic relations over them to facilitate their evaluation.

Commonly the information of the case-base is situated (i.e. is based on the context of the system at the time when the case was stored). Therefore, if the arguments are generated directly from the contents of the case-base, an important issue is to determine how to adapt this information to fit to the current context. This adaptation may be performed by using domain specific rules, as it is the approach of the Case-based Reflective Negotiation model, but due to the dynamicity of argumentation domains these rules may become quickly obsolete. In order to avoid the generation of invalid arguments, case adaptation need from an intensive research in this type of argumentation frameworks. In addition, as pointed out in section 2.1, case-base consistency matters must also be considered.

Another interesting capability that CBR provides agents is the ability to learn from the behaviour of their partners. This functionality opens a wide range of potential improvements for argumentation in MAS. The knowledge acquired from past dialogues could be used to perform a strategic argumentation. This would agents to generate and select arguments that would easily persuade other specific agents.

Other open issues are those related with trust and reputation. In multi-agent argumentation frameworks agents not only need to be able to evaluate arguments, but also to assure the truthfulness of the arguments that they receive. Neglecting trust and reputation issues could result in agents being persuaded by false arguments. Therefore, those agents could learn this corrupt information by storing it in their case-bases.

\section{Case-based Guide of the Interaction Protocol}

In MAS, the dialogue between agents can be controlled by interaction protocols. An interaction protocol makes use of the communication language to define the interaction between the agents and specifies at each time which agent can communicate with other and what it is allowed to say. Interaction protocols also need additional rules to govern, for instance, the admissions or withdrawals of new agents into the dialogue, the proposals validity, the fulfilment of the commitments contracted and the dialogue termination.

How CBR can be applied to improve interaction protocols, or infer some of their rules, is an area of research that has received less attention than case-based argument management. One of the few contributions has been proposed in the AMAL framework, whose agents use a case-based protocol to interact. At each round, an agent of the framework can either assert an argument or rebut it with a counterargument (or a counterexample). If all agents arrive to a consensus in the joint prediction, the interaction protocol ends, otherwise a weighted vote chooses the final solution. Note that both the assertion and evaluation of arguments are determined by the information drawn from the agents' casebase. Therefore, the next move that agents play is based on the decisions taken by the CBR cycle of each 
agent. A different contribution on case-based inference of termination rules has been developed by the Case-based Reflective Negotiation model, whose negotiation strategy determines the maximum time that an agent has before the objective moves to other area.

However, these are preliminary attempts of using a case-based approach to manage the interaction between agents. Future work must investigate how CBR can make further use of the knowledge acquired from past dialogues to guide the interaction between agents. For example, when deciding under which conditions the interaction must finish, the maximum time to interact could be delimited using the information of past dialogues that ended in disagreement due to their excessive duration. Similarly, the necessary time to reach an agreement could be inferred from the time that took to reach past similar agreements.

Finally, some rules over the CBR reasoning cycle can also avoid infinite interactions. AMAL framework has made a first step in addressing this challenge by forbidding agents to send the same argument or counterargument to the same agent during the dialogue. In addition, CBR can also be used to warrant the argumentation success by stopping or readdressing current interactions when they are very similar to past experiences that ended in an unprofitable agreement.

\section{Conclusions}

Recently, the application of Argumentation Theory techniques to assist dialogues in MAS has gained an increasing interest. In this area, there are still many issues to deal with. The aim of this paper has been to clarify the current contributions of CBR to the development of the argumentation in MAS, to identify open issues and to provide new ideas to face future challenges. Storing in cases the information related to past argumentation processes provides a series of interesting advantages for arguments and dialogue management. This article has performed an analysis of how CBR can assist argumentation in MAS. The list below summarises the main advantages that have been identified by this analysis:

- Reasoning with knowledge that has been stored in the form of cases facilitates later revisions and updates when new knowledge is acquired.

- CBR allows agents to learn from past dialogues and thus, improve their autonomy and argumentation skills.

- CBR can be used to generate, select or evaluate arguments.

- If a knowledge-intensive approach for storing the information about arguments in cases is followed, agents are able to reasoning about semantic criteria instead of only reasoning about the syntactic properties of the argument structure.

- The semantic relations over arguments can facilitate their evaluation.

- The CBR explanation techniques can be applied to generate and evaluate arguments in MAS: if explanations are provided, argument evaluation can be performed by checking if the reasoning process that generated an argument was correct.

- CBR can be useful to store and manage the commitments that agents contract through the dialogue:

- The CBR cycle can be used to decide if an agent should contract a commitment that could come into conflict with previous commitments.

- Reasoning about past commitments can determine, in view of the final results obtained from their fulfilment, if contracting a similar commitment is really worth.

- CBR can be use to provide warrants about the agent capability to make a commitment by showing similar commitments that it kept in the past.

- CBR provides agents with the ability to learn from the behaviour of their partners and thus, perform a strategic argumentation: this allows agents to generate and select arguments to persuade other specific agents easily.

- CBR can be applied to improve interaction protocols, or infer some of their rules.

- The maximum time to interact could be delimited using the information of past dialogues that ended in disagreement due to their excessive duration.

- The necessary time to reach an agreement could be inferred from the time that took to reach past similar agreements.

- Some rules over the $\mathrm{CBR}$ reasoning cycle can also avoid infinite dialogues. 
- CBR can also be used to warrant the argumentation success by stopping or readdressing current interactions when they are very similar to past experiences that ended in an unprofitable agreement.

This list is not comprehensive and more advantages can be added as future implementations will appear. Furthermore, the application of CBR to argumentation-based dialogues in MAS is still in its early stages. Currently, there are common assumptions about the agents of the proposed frameworks that could no longer apply in real scenarios. Usually, the agents are thought to be always cooperative, to be reliable, to share the same ontology and to act rationally. These features could not reflect the behaviour of agents in open and real environments. More research must be done to adapt argumentation in MAS to interested agents or heterogeneous agents coming from other platforms. Therefore, CBR must be able to deal with information from different domains and uncertain veracity. This heterogeneity also poses the question about how to manage privacy and security issues in case-bases.

A major advantage of using CBR for argumentation in MAS is that agents can learn from past dialogues by storing in their case-bases the knowledge acquired during the interaction. There is many work to do in order to determine which type of information is useful to store, how to avoid redundancy or how to deal with huge case-bases that could reduce the efficiency of the system.

\section{Acknowledgement}

This work was partially supported by CONSOLIDER-INGENIO 2010 under grant CSD2007-00022 and by the Spanish government and FEDER funds under CICYT TIN2005-03395 and TIN2006-14630-C0301 projects.

\section{References}

Aamodt, A., and Plaza, E. (1994) 'Case-based reasoning; Foundational issues, methodological variations, and system approaches', AI Communications, Vol. 7, No. 1, pp. 39-59.

Aamodt, A. (2004) 'Knowledge-intensive case-based reasoning in Creek', in Proceedings of the 7th European Conference (ECCBR-04), pp. 1-15.

Aleven, V., and Ashley, K.D. (1997) 'Teaching Case-Based Argumentation Through a Model and Examples, Empirical Evaluation of an Intelligent Learning Environment', in Proceedings of the 8th World Conference of the Artificial Intelligence in Education Society, pp. 87-94.

Armengol, E., and Plaza, E. (2001) 'Lazy induction of descriptions for relational case-based learning', in Proceedings of the 12th European Conference on Machine Learning (ECML-01), pp. 13-24.

Ashley, K.D., (1991) 'Reasoning with Cases and Hypotheticals in Hypo', International Journal of Man-Machine Studies, Vol. 34, pp. 753-796.

Aulines, M., Tolchinsky, P., Turon, C., Poch, M., and Cortés, U. (2007), 'Is my spill environmentally safe? towards an integrated management of wastewater in a river basin using agents that can argue', in the 7th International IWA Symposium on Systems Analysis and Integrated Assessment in Water Management.

Bench-Capon, T.J.M., Dunne, P.E. (2007), 'Argumentation in Artificial Intelligence', Artificial Intelligence, Vol. 171, pp. 10-15.

Karacapilidis, N.I., Trousse, B., and Papadias, D. (1997) 'Using Case-Based Reasoning for Argumentation with Multiple Viewpoints', in Proceedings of the 2nd Int. Conference on Case-Based Reasoning (ICCBR-97), pp. 541-552.

Karacapilidis, N., and Papadias, D. (2001) 'Computer supported argumentation and collaborative decision-making: the HERMES system', Information Systems, Vol. 26, No. 4, pp. 259-77.

Modgil, S., Tolchinsky, P., and Cortés, U. (2005) 'Towards formalising agent argumentation over the viability of human organs for transplantation', in Advances in Artificial Intelligence, 4th Mexican International Conference on Artificial Intelligence (MICAI-05), pp. 928-938.

Ontañón, S., and Plaza, E., (2007) 'Learning and Joint Deliberation through Argumentation in Multi-Agent Systems', in Proceedings of the International Conference on Autonomous Agents and Multi-agent Systems (AAMAS-07).

Parsons, S., Sierra, C., and Jennings, N. (1998) 'Agents that reason and negotiate by arguing', Journal of Logic and Computation, Vol. 8, No. 3, pp. 261-292.

Rahwan, I., Ramchurn, S., Jennings, N., McBurney, P., Parsons, S., and Sonenberg, L. (2003) 'Argumentation-Based Negotiation', The Knowledge Engineering Review, Vol. 18, No. 4, pp. 343-375.

Rahwan, I. (2005) 'Guest Editorial: Argumentation in Multi-Agent Systems', Autonomous Agents and Multi-Agent Systems, Vol. 11, No. 2, pp. 115-125. 
Rissland, E.L., Skalak, D.B., and Friedman, M. (1993) 'BankXX: a program to generate argument through case-based search', in Proceedings of the International Conference on Artificial Intelligence and Law (ICAIL-93), pp $117-124$.

Soh, L-K., and Tsatsoulis, C. (2001) 'Agent-Based Argumentative Negotiations with Case-Based Reasoning', AAAI Fall Symposium on Negotiation Methods for Autonomous Cooperative Systems, pp. 16-25.

Soh, L-K., and Tsatsoulis, C. (2005) 'A Real-Time Negotiation Model and a Multi-Agent Sensor Network Implementation', Journal of Autonomous Agents and Multi-Agent Systems, Vol. 11, No. 3, pp. 215-271.

Sørmo, F., Cassens, J., and Aamodt, A. (2005) 'Explanation in Case-Based Reasoning; Perspectives and Goals', Artificial Intelligence Review, Vol. 24, No. 2, pp. 109-143.

Sycara, K. (1990) 'Persuasive argumentation in negotiation', Theory and Decision, Vol. 28.

Tolchinsky, P., Atkinson, K., McBurney, P., Modgil, S., and Cortés, U. (2007) 'Agents Deliberating Over Action Proposals Using the ProCLAIM Model', in the 5th International Central and Eastern European Conference on Multi-Agent Systems (CEEMAS-07).

Tolchinsky, P., Modgil, S., Cortés, U., and Sànchez-Marrè, M. (2006) 'CBR and Argument Schemes for Collaborative Decision Making', in Proceedings of the Conference on Computational Models of Argument (COMMA-06), Vol. 144, pp 71-82.

Tolchinsky, P., Modgil, S., and Cortés, U. (2006b) 'Argument schemes and critical questions for heterogeneous agents to argue over the viability of a human organ', in AAAI Spring Symposium Series; Argumentation for Consumers of Healthcare.

Vázquez-Salceda, J., Cortés, U., Padget, J., López-Navidad, A., and Caballero, F. (2003) 'The Organ Allocation Process: A Natural Extension of the Carrel Agent-Mediated Electronic Institution', AI Communications, Vol. 16, No. 3, pp. 153-165.

Walton, D.N., and Krabbe, E.C.W. (1995) 'Commitment in Dialogue: Basic Concepts of Interpersonal Reasoning', SUNY Press, Albany, NY, USA.

Walton, D. N. (1996) 'Argumentation Schemes for Presumptive Reasoning', Lawrence Erlbaum Associates, Mahwah, NJ, USA. 